\title{
Questes
}

vestes Revue pluridisciplinaire d'études médiévales

$43 \mid 2021$

Domestiquer la nature

\section{Le lis, de la plante domestique à la fleur des rois}

\section{Audrey Dominguez}

\section{(2) OpenEdition}

\section{Journals}

\section{Édition électronique}

URL : https://journals.openedition.org/questes/5814

DOI : $10.4000 /$ questes.5814

ISSN : 2109-9472

\section{Éditeur}

Les Amis de Questes

\section{Édition imprimée}

Date de publication : 30 juillet 2021

Pagination : 75-88

ISSN : 2102-7188

\section{Référence électronique}

Audrey Dominguez, «Le lis, de la plante domestique à la fleur des rois », Questes [En ligne], 43 | 2021, mis en ligne le 30 octobre 2021, consulté le 10 novembre 2021. URL : http://journals.openedition.org/ questes/5814; DOI : https://doi.org/10.4000/questes.5814 


\title{
Le lis, de la plante domestique à la fleur des rois
}

\author{
Audrey DOMINGUEZ
}

Université Grenoble-Alpes

Domestiquer, domescher, adomestir... : le Moyen Âge ne manque pas de verbes quand il s'agit de comprendre la nature comme œuvre divine et source bienfaitrice si elle est savamment utilisée par les hommes. Nous proposons de penser la domestication de la nature en étudiant le lis et son image comme plante domestique dont les caractéristiques remarquables participent au développement du signe de lis comme fleur des rois.

Pour cela, nous nous appuyons essentiellement sur des ouvrages médiévaux latins traduits en français contenant des savoirs encyclopédiques. Ces ouvrages donnent des informations sur le lis et ses usages. Ils permettent aussi de formuler des hypothèses sur la création des représentations du lis ainsi que sur la structuration d'un signe au Moyen Âge. Plus généralement, ils offrent des témoignages sur les manières d'observer et d'appréhender la nature et donc de la domestiquer.

Dans cette perspective, on peut reprendre la définition de domestication comme un acte délibéré d'appropriation et de transformation d'éléments considérés comme naturels afin d'assurer la commodité humaine. Cet acte passe également par la compréhension intellectuelle des éléments envisagés.

Considérant cela, nous nous interrogerons sur les points suivants. Dans quelles mesures les textes scientifiques souvent attribués à des auctoritates, que nous étudions dans cet article, ont-ils influencé la 
formation des images du lis et leurs intégrations dans la société médiévale ? Comment les méthodes d'observation de la nature imprègnent-elles les structures de la pensée au point de rendre domestique la fleur de lis, dans les sens de «qui appartient à la maison » et « fait partie de la maison du roi ou d'un grand personnage ${ }^{1} »$ ?

\section{Description d'une fleur domestiquée}

Les encyclopédies médiévales se fondent notamment sur des ouvrages datant de l'Antiquité pour concevoir le monde. Bernard Ribémont explique ainsi leur développement :

L'écriture encyclopédique se définissait pour une large part comme un discours sur la nature et sur les moyens de l'appréhender, de la définir, de la discuter, de l'écrire et de l'apprendre : natura et disciplinae en quelque sorte ${ }^{2}$.

Les objectifs de ces ouvrages contenant des savoirs encyclopédiques sont alors de comprendre la nature et les éléments qui la composent, leurs qualités et leurs propriétés pour pouvoir mieux définir les usages et par extension, élaborer des réflexions sur la nature ellemême comme création organisée de l'œuvre divine. Le processus de compréhension implique alors des systèmes de classification structurés suivant des critères précis.

Un des principaux critères dépend de la théorie des humeurs développée par Hippocrate puis par Galien, qui définit si un élément est froid ou chaud et s'il est humide ou $\sec ^{3}$. Le lis a ainsi une nature chaude

\footnotetext{
1 «Domestique », dans Dictionnaire du Moyen Français, Atilf - CNRS et Université de Lorraine. URL : http://www.atilf.fr/dmf/definition/domestique (consulté le 14 décembre 2018).

${ }^{2}$ Bernard Ribémont, Les origines des encyclopédies médiévales. D’Isidore de Séville aux Carolingiens, Paris, Honoré Champion, 2001, p. 202.

${ }^{3}$ Jacques Jouanna, «La théorie des quatre humeurs et des quatre tempéraments dans la tradition latine (Vindicien, Pseudo-Soranos) et une source grecque retrouvée »,
} 
et humide. Cette nature permettrait de lutter contre les maux aux humeurs froides, comme le spécifie Barthélemy l'Anglais dans une version de son De proprietatibus rerum composé en latin en 1247, puis traduit en français par Jean Corbechon en 1372 à la demande de Charles V :

Du lis Plinius [dit] que la racine vault avecques sa fleur en moult de manieres, car quant on la boit elle guerit du mors des serpens et si vault contre la malice des choses froides et du venin. ${ }^{4}$

Un autre critère pour définir une plante est de savoir si elle est privée ou sauvage, c'est-à-dire si elle est cultivée, intégrée dans les propriétés de l'homme, domestiquée ou si elle pousse à l'état naturel, sans l'intervention des êtres humains, dans les bois ou dans la forêt. Une plante domestique peut être déterminée par certains aspects physiques discernés lors d'une observation naturaliste ou par ses usages impliquant des qualités utiles à l'homme. Il s'agit souvent de vertus médicinales.

Le lis domestique, cultivé en jardin, se reconnaît ainsi par sa couleur blanche. Nous rapprochons ce lis du Lilium candidum L., qui est l'espèce de lis blanc que nous connaissons le plus de nos jours. Toutefois, il est peu probable qu'il s'agisse exactement du même lis, car les plantes évoluent et sont souvent modifiées par l'homme. Nous notons tout de même que le lis domestique blanc serait le lis le plus ancré dans notre culture et nos représentations mentales.

Le lis blanc est aussi mentionné dans les Étymologies d'Isidore de Séville écrites au début du VII ${ }^{\mathrm{e}}$ siècle ${ }^{5}$, dans le Capitulaire de Villis établi

Revue des Études Grecques, t. 118, 2005, p. 138-167, URL: https://www.persee.fr /doc/reg_0035-2039_2005_num_118_1_4609 (consulté le 15 décembre 2018).

${ }^{4}$ Barthélemy l'Anglais, Livre des propriétés des choses, Paris, BnF, fr. 22531, fo 296297, URL : http://gallica.bnf.fr/ark:/12148/btv1b9059286p/f596.zoom.r=manuscrit\% 2022531.langFR (consulté le 14 décembre 2018). Nous emploierons toujours cette version dans cet article.

${ }^{5}$ Isidore de Séville, Étymologies. Livre XVII, De l'agriculture, éd. Jacques André, Paris, Les Belles Lettres, 1981, p. 168-169. 
en 795 à la demande de Charlemagne $^{6}$, dans Le Livre des simples médecines de Platearius, auteur et professeur en médecine, au XII ${ }^{\mathrm{e}}$ siècle ${ }^{7}$. D'ailleurs, ce dernier distingue les lis blancs, les lis rouges dont la fleur serait plus jolie que celle d'une troisième catégorie, les lis jaunes. Pierre de Crescens, magistrat et agronome qui écrit au tout début du XIV siècle, note également ces trois couleurs pour le lis dans son ouvrage pratique d'agronomie, le Livre des prouffitz champestres et ruraulx ${ }^{8}$. Barthélemy l'Anglais, s'inscrivant dans une démarche d'encyclopédiste du XIII ${ }^{\mathrm{e}}$ siècle, n'évoque pour sa part que le lis rouge et le lis blanc. Le lis rouge est considéré sans hésitation comme un lis sauvage. Pierre Lieutaghi rapproche ce lis rouge du lis martagon, ou lis des montagnes, à qui il reconnaît «des emplois médicinaux et alimentaires analogues à ceux du lis blanc »durant la période médiévale ${ }^{9}$. L'aspect sauvage de la plante viendrait alors du lieu dans lequel elle pousse, tel que la montagne. Cet aspect ne remettrait pas en doute les vertus de la plante, sans pour autant les valoriser. L'opposition domestique/sauvage attribuée au lis ne déterminerait donc pas toujours les usages médicaux de certaines espèces de plantes. Une plante n'a donc pas forcément à être domestiquée pour présenter des effets positifs selon les savoirs médiévaux.

Plus encore, un même végétal peut se trouver à la frontière conceptuelle du sauvage et du domestique. C'est par exemple le cas du lis jaune. Ce dernier ne semble pas offrir un attrait particulier. Platearius le décrit comme une plante domestique, alors que Pierre de Crescens le présente comme une plante sauvage. Cela n'a rien de contradictoire pour

\footnotetext{
${ }^{6}$ Pierre Lieutaghi, Jardin des savoirs, jardin d'histoire, Salagon-Mane, Les Alpes de lumière, 1992, p. 18.

${ }^{7}$ Matthaeus Platearius, Le Livre des simples médecines, éd. Paul Dorveaux, Paris, Société française de l'histoire de la médecine, 1913, p. 103-104.

${ }^{8}$ Pierre de Crescens, Livre des prouffitz champestres et ruraulx, Paris, BnF, Réserve des livres rares, RES-S-286, f ${ }^{\circ}$ 77r-v. URL : https://gallica.bnf.fr/ark:/12148/ btv1b86095128/f173.image (consulté le 14 décembre 2018).

${ }^{9}$ Pierre Lieutaghi, Jardin des savoirs, jardin d'histoire, op. cit., p. 99.
} 
deux raisons. La première raison relève des textes en jeu : il est possible que la traduction en moyen français de Platearius soit corrompue et que le texte de Pierre de Crescens contienne des éléments plus précis et exhaustifs, reprenant directement le texte de Pline. La seconde raison relève davantage de la logique et de la connaissance de la culture des plantes : un lis issu de la nature peut en effet se trouver dans un jardin. Ces éléments montrent ainsi une réflexion relativement incertaine sur le lieu principal dans lequel le lis pousse, et donc sur la définition même du lis jaune, qui peut être confondu notamment avec un lis des marais aussi appelé faux acore durant le Moyen Âge ou l'iris jaune. Nous concluons que le critère de la couleur d'une plante n'est pas toujours suffisant pour déterminer si une plante est domestique ou non.

D'après Barthélemy l'Anglais, les lis domestiques blancs sont ceux qui possèdent le plus de vertus. Ce point pourrait être un constat d'une étude naturaliste : la couleur blanche de la fleur contraste avec la couleur dorée des étamines. Ce contraste visuel remarquable inviterait à penser que la fleur a des vertus médicinales tout aussi impressionnantes. On peut également intégrer d'autres observations naturalistes étonnantes comme la haute tige du lis d'environ 1, 30 mètre qui s'élève vers le ciel ou encore le bon parfum de la fleur qui touche l'odorat et l'esprit du croyant. Geneviève Sodigné-Costes explique notamment que la présence des simples, et donc du lis, dans les jardins d'agréments pour leurs belles couleurs et leur bonne odeur participe à l'instauration d'une atmosphère propice à la contemplation ou la méditation en extérieur ${ }^{10}$.

Cependant, les descriptions naturalistes du lis peuvent également correspondre à des déductions issues de réflexions symboliques. Par

\footnotetext{
${ }^{10}$ Geneviève Sodigné-Costes, «Les simples et les jardins », dans Vergers et jardins dans l'univers médiéval, Aix-en-Provence, Publications du CUERMA, coll. « Senefiance », 1990, p. 336.
} 
exemple, la couleur blanche représente souvent des vertus comme la pureté, ainsi que Michel Pastoureau le souligne ${ }^{11}$. Aussi la culture du lis blanc aurait-elle été favorisée par les symboles du fait de sa couleur. Le symbole influencerait donc les choix de domestication. Les qualités des plantes dont les interprétations symboliques seraient positives intimeraient leur domestication. Ces représentations symboliques nourrissent le discours scientifique. Ce discours réactualise et renouvelle alors les symboles mentionnés.

De plus, les vertus médicinales d'une plante peuvent favoriser la culture de celle-ci dans les jardins, et donc sa domestication. Le lis est intégré dans les pratiques agricoles comme plante d'agrément, mais aussi dans les pratiques médicinales comme un simple, dans le sens de «plante médicinale », qui s'oppose aux remèdes composés, complexes et onéreux des apothicaires. La médecine végétale au Moyen Âge implique souvent la mise en place d'un jardin médicinal, l'herbularius, dans lequel on cueille les plantes fraîches, plus efficaces ainsi ${ }^{12}$.

En outre, le lis, comme la rose, fait partie des plantes qui sortent de l'ordinaire dans la pharmacopée médiévale du fait de sa beauté qui devient un argument majeur de domestication et donc d'appropriation par l'homme. Cela est notamment remarquable dans l'ouvrage de Barthélemy l'Anglais ou encore dans le Jardin médicinal d'Antoine Mizauld, composé à la fin du XVI siècle et influencé par la littérature encyclopédique médiévale. Le médecin parisien décrit la rose et le lis comme « un couple de deux fleurs excellentes ${ }^{13} »$. La rose et le lis se

\footnotetext{
${ }^{11}$ Michel Pastoureau et Dominique Simonnet, Les Couleurs expliquées en images. Paris, Éditions du Seuil, 2015, p. 56.

${ }^{12}$ Pierre Lieutaghi, Jardin des savoirs, jardin d'histoire, op. cit., p. 18-19.

13 Antoine Mizauld, Le Jardin médicinal, éd. Jean Durand, Genève, Imprimerie de Jeremie des Planches, 1578, p. 168, URL : http://www.biusante.parisdescartes.fr /histoire/medica/resultats/index.php? $\mathrm{p}=168 \&$ cote $=41573 \times 02 \&$ do $=$ page $\quad$ (consulté le 15 décembre 2018).
} 
reconnaissent par leurs fleurs qui sont considérées comme possédant une très grande beauté. C'est surtout la couleur intense de ses pétales qui fait la beauté du lis. En effet, Michel Pastoureau explique que l'intensité d'une teinte constitue un élément distinctif essentiel pour la représentation symbolique des couleurs ${ }^{14}$. La valeur esthétique du lis est alors un critère qui constituerait un argument de domestication. Il s'agirait aussi de valoriser la beauté de la création divine, symbole de virginité, de fécondité et de souveraineté, pour reprendre l'interprétation de Michel Pastoureau ${ }^{15}$. Nous notons également que le lis fait partie des rares plantes à se distinguer par sa fleur au Moyen Âge. Cette rareté serait dès lors un argument pour préserver, mais aussi exposer, la plante sacralisée dans un jardin protégé par l'homme.

\section{Le lis de Jean Corbechon : une fleur des rois}

Le lis décrit dans les ouvrages encyclopédiques et naturalistes suivant ses critères physiques et ses vertus est une plante avec des connotations positives au Moyen Âge. Elle est devenue un symbole éminemment valorisé dans la culture occidentale et même un emblème de la royauté en France, comme Anne Lombard-Jourdan le démontre dans son ouvrage Fleur de lis et oriflamme ${ }^{16}$. L'usage de la fleur de lis est d'ailleurs remarquable au XII ${ }^{\mathrm{e}}$ siècle, sous le règne de Louis VII. Le roi aurait fait figurer trois fleurs de lys sur sa bannière lors de la deuxième croisade ${ }^{17}$. Les caractéristiques du lis domestique sont ainsi intégrées

\footnotetext{
${ }^{14}$ Michel Pastoureau et Dominique Simonnet, Les Couleurs expliquées en images, op. cit., p. 60.

${ }^{15}$ Michel Pastoureau, «Une fleur pour le roi. Jalons pour une histoire de la fleur de lis au Moyen Âge », Cahiers du Léopard d'or, vol. 6, 1997, p. 113-130, p. 114.

${ }^{16}$ Anne Lombard-Jourdan, Fleur de lis et oriflamme. Signes célestes du royaume de France, Paris, Presses du CNRS, 1991.

${ }_{17}$ Jean-Marie Pelt, Les Plantes qui guérissent, qui nourrissent, qui décorent, Paris, Chêne, 2014, p. 386.
} 
dans des discours qui ne sont pas directement liés aux enjeux naturalistes. Ils s'en écartent, s'en inspirent pour défendre d'autres causes, comme des causes politiques.

Cela est clair dans la traduction du De Proprietatibus rerum effectuée par Jean Corbechon déjà mentionnée plus tôt. Je voudrais insister sur le dernier paragraphe qui est un ajout de Jean Corbechon mettant en relief le rôle du lis dans la société française. Le traducteur fait alors l'éloge de son pays et du roi français, et tout particulièrement de Charles V qui a commandé la traduction de l'œuvre latine de Barthélemy l'Anglais :

Oultre ce, la fleur de lis a deux prerogatives singuleres sur toutes autres fleurs l'une si est qu'elle est portee aux eglises et mise devant Dieu et devant la Virge Marie par tres grant reverence, l'autre si est que le noble et le plus puissent roy des crestiens, c'est assavoir le gentil et noble roy de France, la porte pour son signe qui lui fut donné et envoié de Dieu le tout puissent et signe de amour et de leauté perpetuelle entre Dieu et les roys de France, laquelle amour et loyauté croist chascum jour et encores croistra, se Dieu plait, sans prandre fin.

D'un point de vue chronologique, Barthélemy l'Anglais n'a pas pu compiler ces références pour Charles V. Le chapitre sur le lis prend une autre tournure avec l'ajout personnel de Jean Corbechon. En effet, ce paragraphe invite à une relecture et à formuler une interprétation dans laquelle le lis domestique observé par les naturalistes permet le développement de discours qui soutiennent la dignité royale française. Cela rejoint d'ailleurs les arguments de Michel Pastoureau, que nous avons mentionnés précédemment :

Toute la propagande royale construite autour des fleurs de lis s'articulera autour de cette idée : le roi de France, responsable du salut de 
ses sujets, a reçu de Dieu une mission. Les fleurs de lis témoignent de cette mission et soulignent le caractère ecclésiastique de la fonction royale ${ }^{18}$.

Certains termes deviennent alors polysémiques. Celui de la fleur peut renvoyer à une partie d'une plante, mais aussi à ce qui est considéré comme le meilleur ou l'essentiel d'un élément à partir du XII ${ }^{\mathrm{e}}$ siècle ${ }^{19}$. La fleur de lis se démarque par sa beauté, ses vertus, en tant que signe religieux. Le roi de France comparé au lis est alors un homme de vertu, «noble», «puissant» et «gentil», un homme reliant les sujets du royaume de France à Dieu. Il devient alors un personnage élu et privilégié, la fleur de la fleur.

Par ailleurs, le verbe croître apparaît deux fois dans ce paragraphe, produisant un effet d'insistance qui renvoie à plusieurs idées. La première idée, explicite, est la volonté de voir se développer et se renforcer le pouvoir du roi français, défenseur de la chrétienté. La seconde idée, plus implicite, serait de comparer ce règne à la croissance du lis, une plante alors remarquable et un signe divin pour le Moyen Âge. D'ailleurs, le lis se démarque par sa haute taille et sa fleur s'érigeant vers le ciel, c'est-dire-vers Dieu. Comparer sa croissance naturelle à celle du développement de la puissance et de la dignité royale inviterait à penser que le règne du roi des Français est un fait naturel et divin, que l'on ne peut pas remettre en question et que l'on se doit d'honorer. Le lis domestiqué, intégré dans les jardins, imprègne aussi les représentations. Il mettrait en forme un autre type de domestication, qui aurait le sens de « soumission, révérence » d'une population humaine, à savoir les sujets du royaume de France. Domestiquer la nature favoriserait en ce sens la fédération des hommes. La nature n'est pas seulement un ornement. Elle

\footnotetext{
${ }^{18}$ Michel Pastoureau, «Une fleur pour le roi », art. cit., p. 118.

19 «Fleur» dans Trésor de la langue française, Atilf - CNRS et Université de Lorraine.
} 
influence l'imaginaire contribuant ainsi à former de nouvelles normes politiques. Cela est d'autant plus évident lorsque l'on considère le contexte socio-politique de la commande de Charles $\mathrm{V}$ cherchant à affirmer son pouvoir troublé dans le cadre de la guerre de Cent $\mathrm{Ans}^{20}$. L'écriture didactique et scientifique ne se détache alors pas du contexte politique et des enjeux de la commande princière. Le savoir naturaliste est alors mis au service de la propagande royale.

Ces interprétations permettent de développer des réflexions plus générales sur le développement des encyclopédies au Moyen Âge, leur rôle et leur fonction. Le développement encyclopédique rappelle notamment l'importance des enjeux politiques et culturels dans l'écriture de textes scientifiques. L'organisation de l'encyclopédie et son contenu sont créés en fonction de celui qui organise les connaissances, de ceux qui, ensuite, les copient et les traduisent, mais aussi du contexte historique plus large. Bernard Ribémont théorise cela :

L'encyclopédie émerge d'une prise de conscience de la nécessité de rassembler un savoir et de l'offrir aux lettrés, dans une période de progrès social obéissant à une dynamique politique ou, pour le moins, de relative stabilité, succédant à ce qui est perçu comme un moment de désorganisation ${ }^{21}$.

La nature intégrerait alors un discours politique, potentiellement en lien avec l'affirmation de nouvelles décisions plus centralisées et bureaucratiques qui doivent se doter d'éléments de théorie politique faisant autorité pour être considérées comme légitimes dans la durée. Parler de la nature et de la nature des choses, c'est alors témoigner de l'époque dans laquelle on vit et raconter les nécessités et les obligations liées à sa culture.

\footnotetext{
${ }^{20}$ Françoise Autrand, Charles V le Sage, Paris, Fayard, 1994.

${ }^{21}$ Bernard Ribémont, Les origines des encyclopédies médiévales, op. cit., p. 203.
} 


\section{Bohort, le chevalier au lis}

Symbole royal, le lis a fortement marqué les mentalités occidentales médiévales. Cela paraît évident dans Le Dit de la fleur de lis de Guillaume de Digulleville, composé en 1338. Cet ouvrage offre des pistes d'explication sur la signification du lis en tant qu'emblème héraldique, en faisant appel à des notions naturalistes. Il a été réédité récemment et la préface de Frédéric Duval donne une analyse très intéressante mettant en lumière la valorisation de la dignité royale grâce à la fleur de lis ${ }^{22}$. Toutefois, nous préférons nous attarder sur un exemple moins évident, dans le but de montrer de quelles façons la description du lis domestiquée s'est imprégnée dans les modes de représentation.

Dans La Queste du Saint Graal, une vision contenant des fleurs de lis présente à Bohort une partie de son destin et de son rôle dans la quête du Graal ${ }^{23}$. Cette vision est expliquée un peu plus tard par un ermite.

Dans ces deux occurrences, les dimensions botaniques et symboliques sont étroitement associées de manière à ce que les savoirs sur la nature produisent un discours moral. Nous notons aussi que le lis n'est mentionné explicitement que dans la première occurrence de la vision alors que dans la seconde, il n'est plus clairement nommé même s'il est sous-entendu lorsque l'auteur parle de flor. Nous devinons le lis grâce à la blancheur de la fleur et grâce à une autre caractéristique botanique: la fleur du lis peut pencher et faire pencher la tige de la plante. Cet aspect, plutôt naturaliste au premier abord, porte dans cet extrait une signification symbolique. La blancheur du lis fait écho à la

\footnotetext{
${ }^{22}$ Guillaume de Digulleville, Le dit de la fleur de lis, éd. Frédéric Duval, Paris, École des Chartes, 2014.

${ }^{23}$ La Quête du Saint Graal, éd. Fanni Bogdanow, Paris, Librairie Générale Française, coll. « Lettres gothiques », 2006, p. 432-434 et p. 464-466.
} 
pureté et à la virginité des femmes, mais aussi à celle des hommes, puisqu'il est question d'éviter le dépucelage d'une jeune fille et d'un chevalier. Ce dépucelage conduirait à la perte de la vertu exprimée dans le texte par la perte de la blancheur de la fleur qui se ternit ou s'efface. De plus, les fleurs penchées, qui ne sont plus tirées vers la hauteur et le ciel, indiquent que des personnes de vertu se détournent de la voie divine et des idéaux moraux et spirituels. Autrement dit, ces personnes ne sont plus «droites comme lis ${ }^{24} »$. Cette expression médiévale, implicite dans $L a$ Queste, nous révèle que les observations naturalistes et la symbolique à laquelle ces observations sont associées inspirent des comportements moraux qui marquent la langue.

Cette imbrication se retrouve aussi lors de l'explication de la vision de Bohort : «que des flors issoit [fueilles et] fruit ${ }^{25}$ ». L'auteur, derrière les mots de l'ermite qui paraît bien connaître la nature, montre que la pucelle et le chevalier évoluent, grandissent, à la manière des plantes, et qu'il leur est possible de devenir des personnes de grande valeur. Cette interprétation paraît s'éloigner du lis, bien qu'il possède un fruit, ou plus exactement une capsule. Ce passage présente des indications botaniques plus générales sur la fleur et le fruit, jouant sur la confusion entre la fleur de lis et la fleur dans le cycle naturel de la plante. Ce dernier est alors mis en correspondance avec le destin de Bohort. Le chevalier erre et doit s'orienter en domestiquant ses passions pour parvenir à la vertu, comme la plante qui pousse et doit être domestiquée afin de donner un lis parfait. Cette mise en parallèle marque ainsi une analogie entre des savoirs botaniques et des considérations morales. Ces considérations morales

24 «Lys », dans Giuseppe Di Stefano, Dictionnaire des locutions en moyen français, Montréal, CERES, 1991, p. 502.

${ }^{25}$ La Quête du Saint Graal, éd. cit., § 229, p. 466. 
sont présentées comme étant en accord parfait avec la nature, donc avec la création divine.

Cette analogie est également visible lorsque le texte aborde la nécessité de prendre soin des fleurs pour éviter qu'elles ne pourrissent. Le verbe pourrir est employé pour désigner la décomposition d'une matière organique. Le pourrissement s'applique à la botanique mais aussi à la médecine. Dans ce dernier cas, la pourriture renvoie à une infection pouvant engendrer la gangrène, la maladie, voire la mort. La pourriture est donc fortement redoutée au Moyen Âge. Le participe passé à valeur adjectivale, pourri, désigne un individu « corrompu, vicieux ${ }^{26} »$ que nous pouvons rattacher à l'explication du rêve. Le pourri représente les personnes de peu de vertu, qui s'éloignent de Dieu. L'observation naturaliste permet à nouveau de donner un sens symbolique et moral qui vise à exposer le rôle du chevalier Bohort. En d'autres termes, la vision de Bohort et son explication par l'ermite mettent en lumière la quête du chevalier et son rôle dans la narration. Bohort doit sauver « les crestiens [...], le cors de honte terriene et 1 'ame de la paine d'enfer ${ }^{27} \gg$ d'après l'explication de l'ermite. Il est alors le protecteur des vertus des Chrétiens luttant contre les pêchés redoutables, notamment celui de la chair. Vertueux et honorable comme une fleur de lis, Bohort est désigné comme le «sergent Jhesucrist », soit le «serviteur » ou l' « agent d'exécution ${ }^{28}$ » de Jésus Christ. Bohort a été choisi pour vivre des aventures avec le soutien et la protection divine. En effet, après avoir décidé de sauver la pucelle au péril de son frère, Bohort voit le membre de sa famille sauvé grâce à un miracle divin. En d'autres mots, Bohort sert Dieu, combat pour

\footnotetext{
26 « Pourrir », dans Dictionnaire du Moyen Français, Atilf - CNRS et Université de Lorraine.

${ }^{27}$ La Quête du Saint Graal, éd. cit., § 229, p. 466.

28 «Sergent », dans Dictionnaire du Moyen Français, Atilf - CNRS et Université de Lorraine.
} 
lui en échange de sa protection et de celle de ses proches. Mimant le système féodal, Bohort doit servir Dieu, comme un chevalier doit servir un roi. Cela fait de lui un personnage rattaché à l'idéal chevaleresque médiéval, à l'instar de Perceval et surtout de Galaad, également comparé à la respectable fleur de lis dans La Queste.

La domestication, ou culture, du lis ne rend pas uniquement compte des connaissances techniques et scientifiques appliquées en médecine ou en agriculture. Elle permet de réfléchir sur la nature des choses, sur leur organisation, ainsi que de légitimer des pouvoirs ou des représentations morales de la société et du monde. Elle contribue à la formation d'images comme celle du bon roi ou encore celle du bon chevalier. Ces figures symboliques se construisent alors sur des savoirs botaniques. Le microcosme du lis donnerait des pistes de réflexions sur le macrocosme, l'entièreté de l'œuvre divine. Ces pistes de réflexion contribuent au développement de mythes qui sont intégrés dans les textes scientifiques, dans les discours politiques ainsi que dans la fiction. Ces discours et histoires fondamentaux invitent à essayer de comprendre une plante, mais aussi de comprendre l'homme, ce qui fait sa vertu, ce qui le nourrit, ce qui le forme et le grandit. 